\title{
Sensory deficits and mental disorders of old age: causal or coincidental associations ? ${ }^{1}$
}

\section{PREVALENCE OF SENSORY DEFICITS AND MENTAL DISORDERS}

\section{General considerations}

The description of concurrent sensory deficits and mental disorder in old age is complicated by general problems in the estimation of the prevalence of morbidity in old age. First, although there is agreement that the onset of 'old age' is some time in the seventh decade of life (i.e. between 60 and 70 years of age), there is no upper age limit. This has the effect of creating an age group which may span 40 or more years. 'Over $65^{\prime}$ 'prevalence figures will consistently underestimate the prevalence of age-related disorders in the oldest age groups. Secondly, criteria for morbidity are not held constant across age groups. In recognition of normative age changes (i.e. changes which appear to occur as a product of ageing, in the absence of pathogens), the standard for 'normal' as opposed to 'abnormal' or 'disease state' is shifted. Higher thresholds for sensation and lower levels of function are frequently described as relative to age normative standards which assume some decrement. Again, this has the effect of underestimating the prevalence of dysfunction in the oldest age groups. The prevalence of sensory deficit is particularly vulnerable to underestimate, as the incidence of disorder increases with age, and criteria for function in the normal range are lowered. As an example, a hearing loss in the range of $25-35 \mathrm{~dB}$ within the speech frequencies, and up to $45-50 \mathrm{~dB}$ at high frequencies, may be considered 'normal' for an 80 year old, although these would constitute a clinically significant hearing loss for a 40 year old.

Age-related losses in sensitivity and acuity of the senses taste, smell and touch have been demonstrated by cross-sectional studies comparing thresholds and discrimination ability of young and old age groups. Although the age differences found in psychophysical studies have been consistently statistically significant (Kenshalo, 1977; Schiffman, 1979), and there have been suggestions that such losses may contribute to anhedonia in old age (Corso, 1981), there has been no serious consideration of their role in the development or exacerbation of mental illness in old age. More attention has been directed to the senses critical to social interaction, orientation and independence: vision and hearing. The prevalence of both vision and hearing impairment increases markedly with age.

\section{Prevalence of vision and hearing impairment}

According to the 1966 US survey conducted by the National Society for the Prevention of Blindness (Fozard et al. 1977), rates of 'legal blindness' across age groups were: 250 per $100000(0.25 \%)$ in the age range 40-64 years; 500 per $100000(0.5 \%)$ in the age range $65-69$ years; and 1450 per $100000(1.5 \%)$ for ages 70 years and over. Likewise, figures from the US National Health Survey (1968) and the Duke Longitudinal Study (Anderson \& Palmore, 1974) indicate an increase in the prevalence of impaired visual acuity in older age groups. Visual acuity poorer than $20 / 50$ in the better eye was found in approximately $10 \%$ of $60-69$ year olds, about $30 \%$ of $70-79$ year olds, and $35 \%$ of those over the age of 80 . Although presbyopic changes are common (i.e. thickening and yellowing of the lens, reduction in the speed and degree of dark adaption, etc.), most severe vision problems in old age are attributable to eye disease not directly related to the ageing process. The

\footnotetext{
1 Address for correspondence: Dr M. R. Eastwood, Clarke Institute of Psychiatry, 250 College Street, Toronto, Ontario M5T 1R8, Canada
} 
four most commonly identified eye pathologies in old age are: (1) macular degeneration; (2) cataract; (3) glaucoma; and (4) diabetic retinopathy (Fozard et al. 1977).

'Deafness', that is, profound bilateral hearing loss which precludes verbal communication and the use of auditory cues in the environment, is relatively rare. Bilateral hearing loss or unilateral losses of sufficient severity to interfere with communication, however, is not uncommon. A US Department of Health, Education and Welfare report (1969) estimated that between 6 and 10 million individuals in the USA (approximately $5 \%$ of the population) are socially handicapped by hearing impairment. Significant hearing loss is more common with advancing age, due to both presbycusic changes and the accumulation of hearing impairments from other causes over the life span.

Based on survey data taken from questionnaires and clinicians' judgements, estimates of the prevalence of hearing impairment among the elderly (i.e. over the age of 65) living in the community are generally in the range of $30-40 \%$. Surveys which include audiometric assessment, however, indicate that closer to $60 \%$ of those living at home or in homes for the aged, and up to 80 or $90 \%$ of the elderly in chronic care facilities, have significant hearing loss (Alpiner, 1978; Gilhome-Herbst \& Humphrey, 1980; Corbin et al. 1984).

\section{Prevalence of psychiatric illnesses in old age}

There is little evidence that the incidence of most mental disorders, unlike sensory deficits, increases with age. Although major affective disorders, neuroses and personality disorders, and even schizophrenia have been noted to manifest for the first time in old age (Kay \& Bergmann, 1980; Gurland \& Cross, 1982), only the class of mental disorders described as organic brain syndromes occurs more commonly in late life. Nevertheless, the accumulation of disorders and the addition of organic brain syndromes do effect a major burden on the mental health of the elderly. By the age of 80 , an estimated $30 \%$ have some form of mental illness. Severe cognitive impairment affects between 3 and $5 \%$ of those over the age of 65 ; at least $10 \%$ have some form of mood disorder; another 5-15\% exhibit a neurosis or personality disorder, or show evidence of substance abuse or dependency; and 1-3\% exhibit paranoid psychoses (Eastwood \& Corbin, 1985).

\section{Coincidence of sensory deficits and psychiatric illness}

There is very little evidence regarding the coincidence of sensory deficits and mental disorder in old age. An exception is the survey conducted by Gilhome-Herbst \& Humphrey (1980), investigating the coincidence of hearing impairment, symptoms of depression and evidence of cognitive impairment in a sample of the elderly living in the community. While this study did not provide psychiatric diagnoses, $60 \%$ of the sample met criteria for clinically significant hearing loss (HL) (defined by pure tone averages greater than $35 \mathrm{~dB}$ HL over the speech frequencies) of which $53 \%$ had depressive symptoms and $20 \%$ had evidence of cognitive impairment, as measured by scales from the Comprehensive Assessment and Referral Evaluation schedule (CARE) (Gurland et al. 1977).

In our recent study of a home for the aged population $66 \%$ of the sample had a hearing impairment by the criterion of weighted pure tone average threshold greater than $35 \mathrm{~dB} \mathrm{HL} ; 53 \%$ had a diagnosable psychiatric disorder (using ICD-9 criteria); and $36 \%$ had both a hearing loss and psychiatric disorder (Eastwood et al. 1985).

\section{EMPIRICAL EVIDENCE OF AN ASSOCIATION}

Evidence regarding an association between acquired sensory deficits and mental disorders in old age comes from clinical observation of geriatric psychiatric patients, comparisons between geriatric psychiatric patients of different diagnostic groups, and from hearing clinic and general population surveys. Perhaps less relevant, but also sometimes cited, are the results of experiments in which sensory deficits were simulated in studies with young subjects. 


\section{Clinical observations}

Several authors have noted what appears to be an excess of sensory deficit in series of geriatric psychiatry patients. Post (1966), in his treatment of persistent persecutory states of the elderly, described an excess of both vision and hearing impairments among these patients. Mayer-Gross et al. (1969) suggested that hearing impairment, because of its greater effect on social interaction, has a more profound psychological consequence. They also described paraphrenics as more likely to suffer from both, and individuals with organic brain syndrome to exhibit hearing loss more often than could be accounted for by advanced age alone.

Clinical audiologists and psychologists working with hearing impaired populations have also described a tendency to withdraw from social interaction and suspiciousness in reaction to hearing loss (Alpiner, 1978; Corso, 1981). Alberti (1977), in detailing problems with provision of aural rehabilitation services in nursing homes and homes for the aged, noted high rates of cognitive impairment, visual and motor handicaps among the hearing impaired in this population.

\section{Clinical comparisons}

Kay \& Roth (1961), in their examination of factors associated with paraphrenia, noted a significantly higher proportion of cases with hearing impairment than was found in other diagnostic categories. Cooper et al. (1974), comparing elderly patients with paranoid and affective psychoses, described overall differences in rates for hearing impairment, and more frequently hearing loss with a conductive component, among the patients with paranoid illness. Although the determination of onset of hearing loss was retrospective, and not precisely dated, the conductive component and histories of middle ear disease appeared to confirm a longer duration of sensory deficit among patients with paranoid illnesses (Cooper \& Curry 1976). In some cases, the hearing loss occurred more than 30 years prior to the onset of psychiatric illness.

In a similar review, Cooper \& Porter (1976) found evidence of more common ocular pathology, chiefly cataract, in their series of paranoid psychotics than among the cases with affective disorders. Although visual acuity in the better eye did not discriminate between the two groups, they reported a higher coincidence of hearing and vision impairment in the paranoid group than in the group with affective disorder. Discussing these findings, Cooper (1976) suggested that individuals with a relatively weak genetic predisposition for schizophrenia may resist the onset of illness until subjected to an accumulation of isolating circumstances (e.g. death, separation, or loss of mobility, vision or hearing).

\section{Surveys}

Moore (1981) reported a survey of the item sheets and case records from 340 psychiatric patients aged 60 years and over who were admitted to the Bethlem Royal and Maudsley Hospitals during 1970-2. Based on the registrars' clinical assessments, only 48 cases were noted to have a sensory deficit, 22 patients with hearing loss, 12 with vision loss and 14 with both. Moore noted that those with recorded sensory deficits were significantly older than those without, and that symptoms of delusions of persecution and organic brain syndrome were also associated with age. Hearing loss was found to be associated with fewer than expected cases of delusions of guilt and affective psychoses, and was recorded for only $30 \%$ of those with paranoid psychoses.

Given the difficulties of evaluating hearing on the basis of either self-report or physician's observation (Corbin et al. 1984), it seems likely that more moderate hearing losses would have been missed by Moore's methodology. As Mayer-Gross et al. (1969) noted, moderate levels of hearing loss may be those which lead to misinterpretation without awareness of the source of difficulty. These authors suggested that, if the hearing loss progresses to a stage of profound deafness, paranoid symptoms may subside. Therefore, Moore's results may have been affected by his focus on more evident cases of sensory deficit.

In the study cited above by Gilhome-Herbst \& Humphrey (1980), the investigators attempted to study the association between sensory deficit and psychiatric symptoms in a sample aged 70 years 
and over and still living in their own homes. Examining the correlations between the degree of hearing loss and evidence of depression or cognitive impairment, they found significant associations, with both more depressive symptoms and greater cognitive impairment being associated with a greater degree of hearing impairment. Both hearing impairment and cognitive impairment were also, however, correlated with advanced age. When the effect of age was factored out, Gilhome-Herbst \& Humphrey reported that the association between cognitive impairment and the degree of hearing loss was no longer significant, although the correlation between hearing loss and depressive symptoms remained so. The authors interpreted these findings as indicating that depressive affect and sensory loss may be causally related, but that the relationship between cognitive and hearing impairment is due to the increased prevalence of both in advanced age.

Norris \& Cunningham (1981), examining the relationship between presbycusic hearing loss and social factors, found no evidence of a correlation between the degree of hearing loss and either the number of social roles or the amount of social participation. Similarly, Thomas et al. (1983), in a study of 239 healthy elderly persons living in the community, found no evidence of increased emotional distress or a reduction in social activity with decreased hearing. They did find decrements in performance on some components of their cognitive test battery, in agreement with previous findings regarding the cognitive test performance of elderly persons with hearing and vision impairments (O'Neil \& Calhoun, 1975; Snyder et al. 1976; Ohta et al. 1981). However, these decrements were restricted to verbal tests and were attributed to a failure to hear questions properly and a reduction in auditory feedback from responses.

In our own study of a sample of 102 residents of a home for the aged, unselected for either psychiatric illness or sensory deficit (Eastwood et al. 1985), we found that 53\% had diagnosable mental disorders, the majority of which were organic brain syndromes. We identified only 14 cases with functional diagnoses, a number too small for the statistical analysis of the degree of sensory loss by diagnosis. However, there were interesting patterns. Five of the six cases with paraphrenia had moderate to severe hearing loss, as did both of the cases with depressive neuroses. In contrast, the three cases with affective psychoses and the three with other neuroses or personality disorder did not have a significant hearing loss. Although these numbers would not support generalization, the pattern of findings with respect to paranoid and affective psychoses, and depressive neuroses, did agree with previous findings (e.g. Cooper et al. 1974; Gilhome-Herbst \& Humphrey, 1980).

Our findings with respect to cases with organic brain syndrome were based on 40 residents. Neither diagnosis of organic brain syndrome nor the degree of cognitive impairment, as measured by the Mini-Mental State Examination (MMSE) (Folstein et al. 1975), was correlated with the presence or degree of hearing impairment. We had assumed that performance on some measures of cognitive function might suffer due to communication problems. To reduce this potential, we used an amplifier and large print materials in interviews with the sensory impaired.

A few reports of psychiatric investigation of patients with primary diagnoses of sensory deficit have been published; however, the elderly have been under-represented in these reports. Singerman et al. (1980) reported an association between psychiatric morbidity, as measured by the General Health Questionnaire (GHQ) (Goldberg, 1978), and objective measures of hearing loss in a series of patients seen in a hearing clinic. The mean ages of their groups with hearing loss, however, ranged from 48 to 56 years. In addition, the group which included the highest proportion of GHQ scores indicating probable psychopathology was that with no evidence of hearing loss.

\section{Simulation experiments}

Work conducted at McGill University in the 1950s by Hebb and his associates (Hebb et al. 1954) established that total sensory deprivation, even for relatively short periods of time, can have psychological effects for some individuals. A simple reduction in hearing sensitivity experienced by students wearing petroleum-impregnated cotton ear plugs (approximately a $30-40 \mathrm{~dB}$ threshold shift) appeared to cause irritability and feelings of alienation and inferiority.

More recently, Zimbardo et al. (1981) reported the production of 'experimental paranoia' in a group of subjects by post-hypnotic suggestions of inability to understand conversation between 
the experimenter and a confederate, thought by the subjects to be other participants in a study on hypnosis. On indices of paranoia (i.e. MMPI items, a clinically derived scale, and both self and independent ratings), the subjects in the experimental condition scored significantly higher than control subjects who were given other post-hypnotic suggestions.

A shortcoming of simulation studies of sensory deficit, in terms of their implications for psychological effects of sensory losses in old age, is that they differ from the late life experience in significant respects. First, simulated losses are of sudden onset, rather than the more common gradual decrement in function. Secondly, in studies which implement devices such as ear plugs, the induced hearing loss is purely conductive and results in a mild to moderate threshold shift across all frequencies. This type of loss is more similar to that sustained when cerumen becomes impacted than the sensorineural losses caused by noise exposure or retrocochlear disease. Finally, as simulation studies have been conducted with young subjects, intact in other respects and fully engaged in the activities of young adults, they suffer from the same lack of relevance for the elderly as do studies of young adults who have lost hearing or vision in war or in other traumatic circumstances, and studies of the congenitally blind or deaf (Thomas, 1981).

\section{CONCLUSIONS}

Given existing evidence, there is little support for a causal relationship between acquired sensory deficit and the mental disorders of old age. The possible exceptions are: first, the onset of non-psychotic depressive symptoms or dysphoria following a sensory loss which is seen by the individual as handicapping or disabling; and, secondly, the catalysis or exacerbation of psychotic illness in individuals with a predisposition to develop schizophrenic illness.

In the first instance, there does appear to be some increase in the report of depressive symptoms with increased sensory deficit, as reported by Gilhome-Herbst \& Humphrey (1980) and by clinicians working with the hearing and visually handicapped. However, this finding has not been universal, nor is sensory deficit necessarily associated with a decrease in social interaction (Norris \& Cunningham, 1981; Thomas et al. 1983). As Thomas (1981) pointed out, the vast majority of individuals with acquired sensory deficits do not develop psychopathology.

In the second instance, as noted by Kay \& Roth (1961) and further elaborated by Cooper et al. $(1974,1976)$ and by Cooper \& Porter (1976), the prevalence of longstanding sensory deficits does appear to be more common in series of cases with paranoid disorders of late life onset than in contrast series of patients with other diagnoses. However, these authors have described sensory deficits as one among many potential risk factors and have suggested that the mechanism is one of reinforcing a pre-existing tendency to social isolation or withdrawal. Perhaps more important than the role which sensory deficits may play in the genesis or exacerbation of paraphrenia is the potential for therapeutic benefit from providing treatment for the sensory impairment. Given that the most frequent ocular pathology identified was cataract (Cooper \& Porter, 1976), which is often operable, and that the most common hearing disorders were those with a conductive component (Cooper et al. 1974, 1976; Eastwood et al. 1985), which often respond to amplification provided by a hearing aid, treatment of the sensory deficit may provide an additional method for managing psychotic symptoms in paraphrenic patients. A case we reported earlier, of a paraphrenic and hearing impaired 75 year old whose psychotic symptoms were dramatically reduced following adjustment to a hearing aid, illustrates this potential (Eastwood et al. 1981).

There is no evidence that sensory deficits per se are risk factors in the aetiology of dementia and depression. However, as sensory deficits are common in old age, and as the development of psychopathology in old age in no way protects from sensory loss, the coincidence of these disorders is high. If, as the evidence suggests, at least half of those who survive into the seventh and eighth decade of life have significant hearing impairment, and at least one quarter a significant impairment of vision, these deficits will, of necessity, affect both the assessment and the management of geriatric psychiatric patients.

In all cases, assessment of mental state by any behavioural measure should be preceded by an 
investigation of sensory function. Evidence of a sensory impairment is an indicator to the clinician to choose methods of assessment which rely less on that sense, to attempt to compensate for the sensory loss by clinical methods (e.g. use of a non-personal amplifier for interviews with hearing impaired individuals who are not wearing a functioning hearing aid), and to take into account the effects of a sensory loss when interpreting findings.

S. L. CORBIN AND M. R. EASTWOOD

\section{REFERENCES}

Alberti, P. W. (1977). Hearing aids and aural rehabilitation in a geriatric population. Journal of Otolaryngology 6, Suppl. 4.

Alpiner, J. G. (1978). Handbook of Adult Rehabilitation Audiology. Williams \& Wilkins: Baltimore.

Anderson, B. \& Palmore, E. (1974). Longitudinal evaluation of ocular function. In Normal Aging (ed. E. Palmore), pp. 24-32. Duke University Press: Durham, N.C.

Cooper, A. F. (1976). Deafness and psychiatric illness. British Journal of Psychiatry 129, 216-226.

Cooper, A. F. \& Curry, A. R. (1976). The pathology of deafness in the paranoid and affective psychoses of later life. Journal of Psychosomatic Research 20, 97-105.

Cooper, A. F. \& Porter, R. (1976). Visual acuity and ocular pathology in the paranoid and affective psychoses of later life. Journal of Psychosomatic Research 20, 107-114.

Cooper, A. F., Kay, D. W. K., Curry, A. R., Garside, R. F. \& Roth, $M$. (1974). Hearing loss in paranoid and affective psychoses of the elderly. Lancet ii, 851-854.

Cooper, A. F., Garside, R. F.\& Kay, D. W. K. (1976). A comparison of deaf and non-deaf patients with paranoid and affective psychoses. British Journal of Psychiatry 129, 532-538.

Corbin, S., Reed, M., Nobbs, H., Eastwood, K. \& Eastwood, M. R. (1984). Hearing assessment in homes for the aged: a comparison of audiometric and self-report methods. Journal of American Geriatrics Soctety 32 (5), 396-400.

Corso, J. (1981). Aging, Sensory Systems and Perception. Praeger: New York.

Eastwood, M. R. \& Corbin, S. (1985). Epidemiology of mental disorders in old age. In Recent Advances in Psychogeriatrics, No. $I$ (ed. T. H. D. Arie). Churchill-Livingstone: London.

Eastwood, M. R., Corbin, S. \& Reed, M. (1981). Hearing impairment and paraphrenia. Journal of Otolaryngology 10(4), 306-308.

Eastwood, M. R., Corbin, S. L., Reed, M., Nobbs, H. \& Kedward, H. B. (1985). Acquired hearing loss and psychiatric illness: an estimate of prevalence and co-morbidity in a geriatric setting. British Journal of Psychiatry (in the press).

Folstein, M. F., Folstein, S. E. \& McHugh, P. R. (1975). 'MiniMental State': a practical method for grading the cognitive state of patients for the clinician. Journal of Psychiatric Research 12, 189-198.

Fozard, J. L., Wolf, E., Bell, B., McFarland, R. A. \& Podolsky, S. (1977). Visual perception and communication. In Handbook of the Psychology of Aging (ed. J. E. Birren and K. W. Schaie), pp 497-534. Van Nostrand: New York.

Gilhome-Herbst, K. \& Humphrey, C. (1980). Hearing impairment and mental state in the elderly living at home. British Medical Journal 281, 903-905.

Goldberg, D. P. (1978). Manual of the General Health Questionnatre. General Practice Research Unit. Maudsley Hospital: London.

Gurland, B. J.\& Cross, P. S.(1982). Epidemiology of psychopathology in old age: Some implications for clinical services. The Psychiatric Clinics of North America 5 (1), 11-26.

Gurland, B. J., Kuriansky, J. B., Sharpe, L., Simon, R., Stiller, P. \& Birkett, P. (1977). The comprehensive assessment and referral evaluation (CARE)-rationale, development and reliability.
International Journal of Aging and Human Development 8 , 9-42.

Hebb, D. O., Heath, E. A. \& Stuart, E. A. (1954). Experimental deafness. Canadian Journal of Psychology 8, 152-156.

Kay, D. W. K. \& Bergmann, K. (1980). Epidemiology of mental disorder among the aged in the community. In Handbook of Mental Health and Ageing (ed. J. E. Birren and R. B. Sloane), pp. 34-56. Prentice-Hall: Englewood Cliffs.

Kay, D. W. K. \& Roth, M. (1961). Environmental and hereditary factors in the schizophrenias of old age ('late paraphrenia') and their bearing on the general problem of causation in schizophrenia. Journal of Mental Science 107, 649-686.

Kenshalo, D. R. (1977). Age changes in touch, vibration, temperature, kinesthesis and pain sensitivity. In Handbook of the Psychology of Aging (ed. J. E. Birren and K. W. Schaie), pp. 562-579. Van Nostrand: New York.

Mayer-Gross, W., Slater, E. \& Roth, M. (1969). Clinical Psychiatry. Baillière, Tindall and Cassell: London.

Moore, N. C. (1981). Is paranoid illness associated with sensory defects in the elderly? Journal of Psychosomatic Research 25(2), 69-74.

National Society for Prevention of Blindness (1966). Estimated statistics on blindness and vision problems. National Society for the Prevention of Blindness: New York.

Norris, M. L. \& Cunningham, D. R. (1981). Social impact of hearing loss in the aged. Journal of Gerontology 36(6), 727-729.

Ohta, R. J., Carlin, M. F. \& Harmon, B. M. (1981) Auditory acuity and performance on the MSQ in the elderly. Journal of the American Geriatrics Society 29(10), 476-478.

O'Neil, P. M. \& Calhoun, R.S. (1975). Sensory deficits and behaviour deterioration in senescence. Journal of Abnormal Psychology 84(5), 579-582.

Post, F. (1966). Persistent Persecutory States of the Elderly. Pergamon Press: London.

Schiffman, S. (1979). Changes in taste and smell with age: Psychophysical aspects. In Sensory Systems and Communication in the Elderly (Aging Vol. 10) (ed. J. M. Ordy and K. Brizzee), pp. 211-243. Raven Press: New York.

Singerman, B., Riedner, E. \& Folstein, M. (1980). Emotional disturbance in hearing clinic patients. British Journal of Psychiarry 137, 58-62.

Snyder, L. N., Pyrek, J. \& Smith, K. C. (1976). Vision and mental functions of the elderly. Gerontologist 16(6), 491-495.

Thomas, A. J. (1981). Acquired deafness and mental health. British Journal of Medical Psychology 54, 219-229.

Thomas, P. D., Hunt, W. C., Garry, P. J., Hood, R. B., Goodwin, J. M. \& Goodwin, J. S. (1983). Hearing acuity in a healthy elderly population: Effects on emotional, cognitive and social status. Journal of Gerontology 38(3), 321-327.

United States Department of Health, Education and Welfare (1969). Human Communication and lts Disorders. Bethesda, Md.

United States National Health Survey (1968). Monocular-Binocular Visual Acuity of Adults. Public Health Service Publication Number 100. Series 11, Number 30, 1960-1962.

Zimbardo, P. G., Anderson, S. M. \& Kabat, L. G. (1981). Induced hearing deficit generates experimental paranoia. Science 212, $1529-1531$. 